\title{
Thoracic Epidural Analgesia
}

National Cancer Institute

\section{Source}

National Cancer Institute. Thoracic Epidural Analgesia. NCI Thesaurus. Code C150574.

Epidural analgesia administered to the thoracic spine to treat pain in the thoracic and upper abdominal areas during surgery. 